\title{
Horizons and Ergoregions in Superfluids
}

\author{
G.E. Volovik \\ Low Temperature Laboratory, Helsinki University of Technology \\ P.O.Box 2200, FIN-02015 HUT, Finland \\ Landau Institute for Theoretical Physics, Kosygina 2, 119334 Moscow, Russia
}

\begin{abstract}
Ripplons - gravity-capillary waves on the free surface of a liquid or at the interfaces between two superfluids - are the most favourable excitations for simulation of the general-relativistic effects related to horizons and ergoregions. The white-hole horizon for the "relativistic" ripplons at the surface of the shallow liquid is easily simulated using the kitchen-bath hydraulic jump. The same white-hole horizon is observed in quantum liquid - superfluid ${ }^{4} \mathrm{He}$. The ergoregion for the "non-relativistic" ripplons is generated in the experiments with two sliding ${ }^{3}$ He superfluids. The common property experienced by all these ripplons is the Miles instability inside the ergoregion or horizon. Because of the universality of the Miles instability, one may expect that it could take place inside the horizon of the astrophysical black holes, if there is a preferred reference frame which comes from the trans-Planckian physics. If this is the case, the black hole would evapotate much faster than due to the Hawking radiation. Hawking radiation from the artificial black hole in terms of the quantum tunneling of phonons and ripplons is also discussed.
\end{abstract}

PACS numbers: 04.70.-s, 67.40.Hf

\section{INTRODUCTION}

Black hole is the region of spacetime in which gravity is so strong that not even light can escape from this region. There are many astrophysical black hole candidates, but the unambiguous observational evidence for the existence of black holes has not yet been established (see, e.g. review papers 1). The defining property of the black hole - the event horizon - is still missing in the experiment, though there are indications that some of the candidates might have event horizons. At the moment we are not able to study experimentally the exotic phenomena related to the event horizon, 


\section{G.E. Volovik}

for example, how the presence of the horizon modifies the properties of the vacuum of relativistic quantum fields. That is why the modeling of the event horizon in condensed matter systems may provide us with some ideas useful for study of the astronomical black holes, their formation and decay. The close analogy between the relativistic quantum vacuum and the ground state of quantum condensed matter appears to be instrumental. In this respect the quantum liquids, such as superfluids, are the best candidates for simulations: the horizon can be relatively easy constructed in the moving liquid, and the properties of the quantum vacuum in the presence of the horizon can be studied.

Two-fluid hydrodynamics of superfluid liquids describes the coherent motion of the superfluid 'quantum vacuum' and dynamics of excitations above the ground state - quasiparticles - which form the normal component of the liquid. At low energy some quasiparticles mimic relativistic particles propagating in the effective curved space-time produced by the distortions of the superfluid vacuum and by its superfluid flow. This is an example of the quantum gravity provided by quantum liquids. Starting from the transPlanckian physics - quantum many body system of interacting atoms - one obtains in the low energy corner the emergent quantum fields of relativistic quasiparticles living in the background of the emergent classical gravity field.

The effective gravity in quantum liquids is dynamical, but as distinct from the Einstein theory of gravity, the metric field typically obeys the hydrodynamic equations rather than Einstein equations. There is an exclusion from this rule: in superfluid ${ }^{3} \mathrm{He}-\mathrm{A}$, the effective quantum electrodynamics of "relativistic" fermions and "photons" emerges at low energy. These quantum fields propagate in effective space-time whose metric field obeys equations remotely resembling Einstein equations ${ }^{2}$. All this occurs because superfluid ${ }^{3} \mathrm{He}-\mathrm{A}$ belongs to the special universality class of Fermi systems.

However, even if the effective gravity in liquids is distinct from the Einstein gravity, it can be useful for simulations of many phenomena related to Einstein gravity, including the physics of black holes. For example, the effect of Hawking radiation is solely determined by the behavior of quantum fields in the vicinity of the event horizon, and it does not matter for which excitations (photons or sound waves) the horizon is constructed and from which equations (Einstein or hydrodynamic) it is obtained.

The acoustic analog of a black hole - the horizon for sound waves has been suggested by Unruh in 1981 ․ The event horizon for sound waves emerges if the flow velocity of the liquid exceeds the local speed of sound $c$ in some region of liquid: sound waves (or quasiparticles in superfluids phonons) cannot escape from this region. The propagation of sound in the presence of the acoustic horizon is described by the so called acoustic metric, 


\section{Horizons and Ergoregions in Superfluids}

which is similar to the metric describing the space-time in general relativity in the presence of the black hole. This is discussed in Sec. 2. together with the analog of the Hawking radiation which is described in terms of quantum tunnelling between the classical trajectories 4156 . Since 1981 there appeared many suggestions to simulate the black hole and white hole horizons for various excitations in various laboratory systems (see review paper ${ }^{\mathbf{7}}$ and references therein).

In Sec. 3. we discuss the most perspective analog - the effective $2+1$ space-time emerging for the surface waves (ripplons) in the shallow water limit. The effective horizon for ripplons has been first suggested by Schutzhold and Unruh 8 . This analog demonstrates the mechanism of the decay of the black hole which is alternative to the Hawking radiation - the instability of the quantum vacuum behind the horizon (Sec. 3.4.). This mechanism is applicable both to the relativistic systems with the horizon and non-relativistic systems with the ergoregion, and it has been experimentally observed for the non-relativistic ripplons propagating at the interface between two superfluids 91011. Similar instability of the vacuum inside the astronomical black hole is possible.

In Sec. 4. we discuss experiments with the hydraulic jump in superfluid ${ }^{4} \mathrm{He} 12$. This circular hydraulic jump simulates the $2+1$ dimensional white hole for the relativistic ripplons and the instability inside the horizon 13 .

In Sec. 5. some perspectives are discussed.

\section{GRAVITY FOR PHONONS}

\subsection{Effective Metric}

In the frame moving with velocity $\mathbf{v}_{\mathrm{s}}$ of the superfluid vacuum - the comoving frame - the spectrum of sound waves is 'relativistic': $\omega^{\text {com }}(\mathbf{k})=$ $\pm c k$, where $c$ is the speed of sound. In the laboratory frame, the spectrum is Doppler shifted:

$$
\omega(\mathbf{k}, \mathbf{r})= \pm c k+\mathbf{k} \cdot \mathbf{v}_{\mathbf{s}}(\mathbf{r})
$$

or

$$
\left(\omega-\mathbf{k} \cdot \mathbf{v}_{\mathrm{s}}\right)^{2}=c^{2} k^{2} .
$$

This can be written in the general Lorentzian form

$$
g^{\mu \nu} k_{\mu} k_{\nu}=0
$$

where $k_{0}=-\omega$, and the contravariant components of the metric are

$$
g^{00}=-1, g^{0 i}(\mathbf{r})=-v_{\mathrm{s}}^{i}(\mathbf{r}), g^{i j}(\mathbf{r})=c^{2} \delta^{i j}-v_{\mathrm{s}}^{i}(\mathbf{r}) v_{\mathrm{s}}^{j}(\mathbf{r}) .
$$




\section{G.E. Volovik}

The inverse matrix $g_{\mu \nu}$ determines the trajectories of phonons in the moving liquid. This covariant metric describes the effective (3+1)-dimensional spacetime in which phonons move along the geodesic lines $d s^{2}=0$ :

$$
d s^{2}=g_{\mu \nu} d x^{\mu} d x^{\nu}=-d t^{2}+c^{-2}\left(d \mathbf{r}-\mathbf{v}_{\mathbf{S}}(\mathbf{r}) d t\right)^{2} .
$$

\subsection{Black and White Holes}

The most instructive for us is the spherically symmetric flow when the superfluid velocity is radial: $\mathbf{v}_{\mathrm{s}}(\mathbf{r})=\hat{\mathbf{r}} v(r)$ :

$$
d s^{2}=-\left(1-v^{2}(r) / c^{2}\right) d t^{2}-2 c^{-2} v(r) d r d t+c^{-2}(d \mathbf{r})^{2} .
$$

The coordinate transformation

$$
\tilde{t}=t+\int^{r} d r \frac{v(r)}{c^{2}-v^{2}(r)} .
$$

leads to the more familiar metric

$$
d s^{2}=-\left(1-\frac{v^{2}(r)}{c^{2}}\right) d \tilde{t}^{2}+\frac{d r^{2}}{c^{2}} \frac{1}{1-\frac{v^{2}(r)}{c^{2}}}+\frac{1}{c^{2}} r^{2} d \Omega^{2} .
$$

In the particular case when the velocity field has the form $v^{2}(r)=$ $2 G M / r$, the equation (8) reproduces the Schwarzschild line element in Einstein gravity, where $M$ is the mass of the gravitating body, and $G$ is the Newton constant. The corresponding equation (6) with $v^{2}(r)=2 G M / r$ is known as the Painlevé-Gullstrand line element which describes the gravity field of the same body but in different coordinate system - the coordinate system adapted to the free falling particle 14 .

In moving superfluids, the Painlevé-Gullstrand type metric (15) with nonzero off-diagonal element $g_{0 i}$ is naturally generated by flow since $g_{0 i} \propto v_{\mathrm{s} i}$. The notion of the black and white event horizons has also a very simple meaning in liquids. For example, let us assume that we are able to construct the spherically symmetric flow with $v(r)<0$ such that within some radius $r<r_{h}$ one has $v^{2}(r)>c^{2}$. Then phonons cannot escape from this region since they are dragged by the superfluid vacuum to the center with the speed faster than their velocity $c$. Thus the surface $r=r_{h}$ at which $v(r)=-c$ would serve as the horizon of the black hole.

On the contrary, if the liquid flows outward, i.e. for $v(r)>0$, all the phonons are necessarily dragged away from the region $r<r_{h}$. In this case, the surface $r=r_{h}$ at which $v(r)=c$ would serve as the horizon of the white hole. 


\section{Horizons and Ergoregions in Superfluids}

Though in the Einstein gravity the Schwarzschild metric and the PainlevéGullstrand metric describe the same gravitational field, the latter is more preferable especially when the physics of the event horizon is concerned. The Schwarzschild metric contains the singularity at the horizon: the $g_{r r}$ element of the metric is infinite at $v^{2}(r)=c^{2}$. This is the so-called coordinate singularity - the non-physical singularity which can be removed by coordinate transformation to the Painlevé-Gullstrand metric. The latter is smooth across the horizon, which allows us to study the behavior of the quantum fields and quantum vacuum both outside and inside the horizon. This is the reason why the Painlevé-Gullstrand metric which provides us with the simple "river" model of black hole popular now in physics of the event horizon (see Ref. ${ }^{15]}$ and references therein).

\subsection{Hawking Radiation as Tunneling}

As distinct form the Einstein equations which admit the spherically symmetric solutions with the horizon, the equations of hydrodynamics do not support the spherically symmetric acoustic horizon. Acoustic horizon occurs in a different geometry: in the so-called Laval nozzle, where the horizon takes place at the narrowest cross section of the tube. Fortunately, the shape of the surface of the horizon is not important for the discussion of the most interesting quantum properties of the quantum fields in the horizon - the Hawking radiation. This effect can be illustrated using the simplest case of the one-dimensional flow with varying velocity $v(x)$ and varying speed of sound $c(x)$. The effective acoustic metric experienced by phonons has the form

$$
d s^{2}=g_{\mu \nu} d x^{\mu} d x^{\nu}=-d t^{2}+\frac{1}{c^{2}(x)}(d x-v(x) d t)^{2},
$$

and the horizon occurs at the point $x=x_{0}$ where $v\left(x_{0}\right)=c\left(x_{0}\right)$, or $v\left(x_{0}\right)=$ $-c\left(x_{0}\right)$. The effective gravitational field at the horizon is given by

$$
g_{x}=\left.(1 / 2) \nabla_{x}\left(c^{2}-v^{2}\right)\right|_{x_{0}} .
$$

Let us choose the flow with $v(x)>0$. To simulate the black hole horizon, we must take $v(x)<c(x)$ at $x<x_{0}$ (exterior region) and $v(x)>$ $c(x)$ at $x>x_{0}$ (region behind the horizon). In the local reference frame comoving with liquid, the energy spectrum of phonons is everywhere positive $\hbar \omega^{\mathrm{com}}(k)=\hbar c k>0$, and thus at $T=0$ phonons are not excited. This vacuum state is, however, not in equilibrium since in the local comoving frame the whole velocity field is time dependent and thus the quasiparticle energy and the vacuum as a whole is not well defined. The energy is well 


\section{G.E. Volovik}

defined in the laboratory frame where the velocity field is time independent. But in this frame some modes acquire negative energy behind the horizon, $\hbar \omega(\mathbf{k})=\hbar c k+\hbar \mathbf{k} \cdot \mathbf{v}_{\mathbf{s}}<0$. All this indicates that the initial comoving quantum vacuum is unstable in the presence of the black hole. Hawking radiation provides the mechanism for the decay of the black hole unless some other, more violent, process of vacuum instability intervenes.

In the semiclassical description, the Hawking radiation can be considered as the quantum tunneling between classical trajectories 4156 (the same analysis applied for the Unruh effect in superfluids see in Ref. 16). We consider the positive frequency modes, $\omega=\omega(k)>0$, as viewed in the laboratory frame. Classical trajectories $k(x)$ of phonons may be found from Eq.(11): $\omega= \pm c(x)|k(x)|+v(x) k(x)$. There are two relevant trajectories in Fig. 1]

$$
k(x)=\frac{\omega}{v(x)-c(x)} .
$$

The trajectory with $k(x)<0$ starts at the horizon and propagates into the exterior region. In the comoving frame the phonon frequency is positive, $\omega^{\mathrm{com}}=c(x)|k(x)|$. Formally the trajectory starts with infinite frequency, $\omega^{\text {com }}\left(x_{0}\right)=+\infty$, but practically there is the cut-off which in quantum liquids is given by the Debye frequency, and in the quantum vacuum - by the Planck energy scale. The trajectory with $k(x)>0$ also starts near horizon but propagates into the region behind the horizon. In the comoving frame the phonon frequency is negative, $\omega^{\operatorname{com}}(k)=-c(x)|k(x)|$, and approaches $-\infty$ at the horizon.

Quantum mechanics allows the phonon to tunnel between the two trajectories. The tunneling exponent is determined by the conventional semiclassical action

$$
S=\operatorname{Im} \int \hbar k(x) d x=\operatorname{Im} \int \frac{\hbar \omega}{v(x)-c(x)} d x,
$$

The momentum $k(x)$ on the classical trajectory has a pole at the horizon

$$
k(x) \approx \frac{\omega c\left(x_{0}\right)}{g_{x}\left(x-x_{0}\right)},
$$

Shifting the contour of integration into the upper half-plane of the complex variable $x$, one obtains for the tunneling action

$$
S=\frac{\pi \hbar \omega c\left(x_{0}\right)}{g_{x}} .
$$

The tunneling between the trajectories describes the process of creation of the pair of phonons, one of which is radiated into the exterior region. The 


\section{Horizons and Ergoregions in Superfluids}

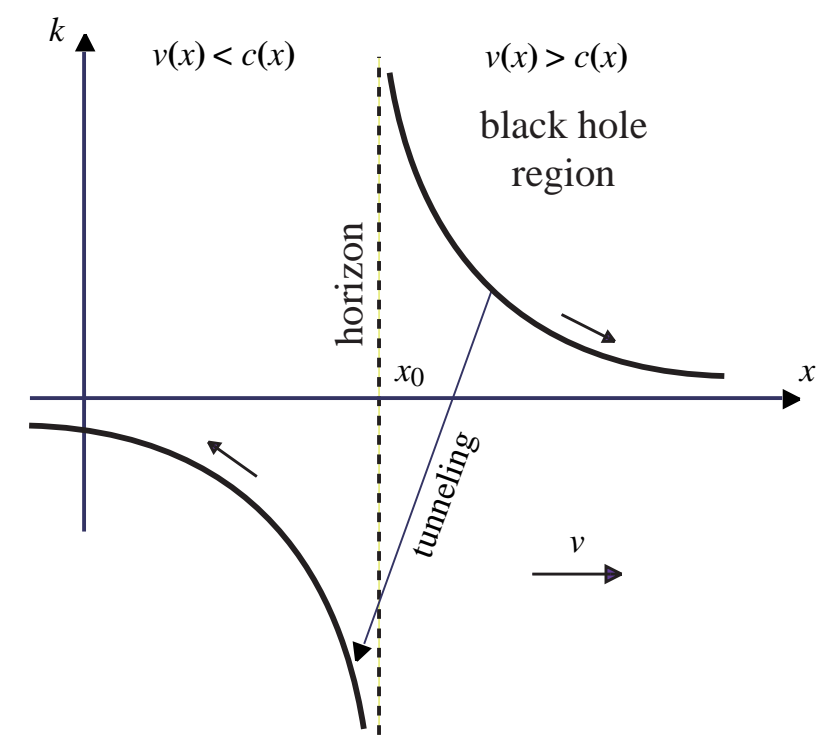

Fig. 1. Two relevant rajectories of massless 'relativistic' quasiparticles phonons - travelling from the horizon of acoustic black hole. Arrows show the direction of motion. Quasiparticles have diverging energy in the vicinity of the horizon (infinite blue shift) which must be cut off by Planckian (Debye) energy scale. The tunneling between the two trajectories describes the process of emission of phonons from the horizon which is analogous to the Hawking radiation from the black hole.

probability of tunneling demonstrates that the radiation from the (acoustic) black hole is thermal with the Hawking temperature $T_{\mathrm{H}}$ being proportional to the (effective) gravity at the horizon:

$$
P \propto \exp \left(-\frac{2 S}{\hbar}\right)=\exp \left(-\frac{\hbar \omega}{T_{\mathrm{H}}}\right), T_{\mathrm{H}}=\frac{\hbar g_{x}}{2 \pi c} .
$$

This semiclassical approach is only valid when the tunneling action is large compared to Planck constant, i.e. when $\hbar \omega \gg T_{\mathrm{H}}$.

The Hawking radiation is completely determined by the (effective) metric in the vicinity of the horizon. It does not depend on the motion equations for the gravity field: the metric can obey Einstein, hydrodynamic or other equations which allow for the existence of an event horizon. The Eq. (15) is applicable for any relativistic excitations radiated from the horizon, including 'relativistic' fermionic quasiparticles living in superfluid ${ }^{3} \mathrm{He}-\mathrm{A}{ }^{175}$.

Note that in the above derivation of the Hawking temperature we actually did not use the full quantum mechanics. Only the wave mechanics was exploited, which is also applicable to the classical waves propagating in 


\section{G.E. Volovik}

the background classical metric. Eq.(15) can be rewritten without using the Planck constant $\hbar$ (see Ref. 18 ):

$$
P \propto \exp \left(-\frac{\omega}{\omega_{\mathrm{H}}}\right), \omega_{\mathrm{H}}=\frac{g_{x}}{2 \pi c} .
$$

The above equation describes the spectrum of classical waves radiated from the horizon. In the full quantum mechanics the radiation is caused by quantum fluctuations in the vicinity of the horizon. But in the wave mechanics the radiation can be generated by some external noise introduced in the vicinity of the horizon or by thermodynamic fluctuations. However, these initial perturbations near the horizon are only responsible for the prefactor in Eq.(16), which was missing in our derivation, but they do not influence the value of the Hawking frequency $\omega_{\mathrm{H}}$. That is why the Hawking frequency can be measured in classical experiments.

\subsection{Does Hawking Radiation Exist?}

Let us mention that in the literature there is a controversy concerning the Hawking radiation. There is even a point of view that a Schwarzschild black hole formed during a collapse process does not radiate (see e.g. Ref.

19). The other authors (see e.g. Ref. ${ }^{20}$ ) suggest that the Hawking radiation may exist but the Hawking temperature is twice larger than that originally calculated by Hawking, i.e. twice that in Eq.(15).

From our point of view the existence of Hawking radiation and the Hawking temperature depend on the vacuum state. In our case when the horizon is formed by the moving superfluid liquid, the initial vacuum is the comoving vacuum, i.e. the vacuum seen in the frame moving with the liquid. The comoving vacuum does not coincide with the equilibrium vacuum determined by the Killing vector energy (the vacuum which is seen in the laboratory frame). That is why the comoving vacuum is unstable, and the initial stage of the development of instability is the Hawking radiation (see discussion in Ref. 2). These two vacua and the quantum tunneling are described in the Painlevé-Gullstrand frame, which is the natural frame for the propagation of quasiparticles in the moving liquids.

On the contrary, the Schwarzschild coordinates are irrelevant for this problem: they are obtained after singular coordinate transformation which is unphysical in superfluids since it removes the part of the physical space. This is the typical situation in condensed matter, that in general the singular coordinate transformations are forbidden. As a result the coordinate frames are distributed into classes: the transition between the classes is only possible after singular ccordinate transformations. For the artificial (acoustic) 


\section{Horizons and Ergoregions in Superfluids}

black holes where the proper frame is the Painlevé-Gullstrand frame and the proper initial vacuum is the comoving vacuum, the Hawking radiation is the real process. The original Hawking's derivation ${ }^{21]}$ has been made in the frame which belongs to the same class as the Painlevé-Gullstrand frame. That is why the radiation from the artificial black hole is characterized by the same temperature as was derived by Hawking. However, the Hawking radiation from the astrophysical black hole stiil remains the open question since the problem of the correct choice of the vacuum state after collapse is not solved.

Note also that for quantum liquids, in some cases the Hawking radiation is suppressed because of the effect which is similar to the Coulomb blockade in mesoscopic physics: the radiated quasiparticle disturbs the vacuum state so that the radiation becomes impossible. For example, the radiated quasiparticle changes the superfluid current and thus violates the mass conservation law. However the radiation of two quasiparticles is possible, since it does not violate the conservation law. The simultaneous tunneling of two quasiparticles is called the co-tunneling. The corresponding tunneling exponent describing the co-tunneling process is twice the tunneling exponent of a single quasiparticle. As a result the Hawking temperature is twice smaller than in Eq. (15), i.e the Coulomb blockade effect is opposite to that suggested in Ref. 20.

\section{GRAVITY FOR RIPPLONS}

In all the condensed matter systems suggested for simulation of gravity, even if the horizon can be constructed in principle, the estimate for the Hawking temperature is very pessimistic for the observation of the Hawking radiation (though the classical analog of the Hawking effect discussed in the previous section can be observed). That is why it is instructive to look for the other possible experimental consequences of the event horizon. The effective gravity for ripplons - the capillary-gravity waves on the free surface of the liquid or at the interface between two liquids - provides us with the new mechanism of the decay of the black hole. Let us start with ripplons propagating on the surface of the liquid.

\subsection{Effective Metric on the Surface of Liquid}

The general dispersion relation $\omega(\mathbf{k})$ for ripplons on the surface of a liquid is

$$
M(k)(\omega-\mathbf{k} \cdot \mathbf{v})^{2}=F+k^{2} \sigma .
$$




\section{G.E. Volovik}

Here $\sigma$ is the surface tension; $F=\rho g$ is the gravity force where $\rho$ is mass density of the liquid; and $\mathbf{v}$ is the velocity of the liquid. The quantity $M(k)$ is the $k$-dependent mass of the liquid which is forced into motion by the oscillating surface:

$$
M(k)=\frac{\rho}{k \tanh k h},
$$

where $h$ is the thicknesses of the layer of the liquid.

The spectrum (17) becomes "relativistic" in the shallow water limit $k h \ll 1, k \ll k_{0}$ :

$$
(\omega-\mathbf{k} \cdot \mathbf{v})^{2}=c^{2} k^{2}+c^{2} k^{4}\left(\frac{1}{k_{0}^{2}}-\frac{1}{3} h^{2}\right) \quad, \quad c^{2}=g h \quad, \quad k_{0}^{2}=\rho g / \sigma .
$$

If the $k^{4}$ corrections are ignored, the spectrum of ripplons is described by the effective $2+1$ metric 8

$$
g^{\mu \nu} k_{\mu} k_{\nu}=0 \quad, \quad k_{\mu}=\left(-\omega, k_{x}, k_{y}\right)
$$

with the following elements

$$
g^{00}=-1, g^{0 i}=-v^{i}, g^{i j}=c^{2} \delta^{i j}-v^{i} v^{j} .
$$

The interval describing the effective $2+1$ space-time in which ripplons propagate along geodesics and the corresponding covariant components of the effective metric are

$$
d s^{2}=g_{\mu \nu} d x^{\mu} d x^{\nu} \quad, \quad g_{00}=-1+\frac{v^{2}}{c^{2}}, g_{0 i}=-\frac{v^{i}}{c^{2}}, g_{i j}=\frac{1}{c^{2}} \delta_{i j} .
$$

\subsection{Effective Metric on the Interface Between Two Superfluids}

The spectrum of ripplons propagating along the interface between two superfluids:

$$
M_{1}(k)\left(\omega-\mathbf{k} \cdot \mathbf{v}_{1}\right)^{2}+M_{2}(k)\left(\omega-\mathbf{k} \cdot \mathbf{v}_{2}\right)^{2}=F+k^{2} \sigma .
$$

Here, as before, $\sigma$ is the surface tension of the interface and $F$ is the force stabilizing the position of the interface. For the interface between ${ }^{3} \mathrm{He}-\mathrm{A}$ and ${ }^{3} \mathrm{He}-\mathrm{B}$ the gravity force is negligible, since these liquids have almost equal densities: $\left|\rho_{1}-\rho_{2}\right| \sim 10^{-5}\left(\rho_{1}+\rho_{2}\right)$. However, these two superfluids have essentially different magnetic properties, and the corresponding force $F$ which stabilizes the position of the interface is provided by the gradient of external magnetic field. The effective masses $M_{1}(k)$ and $M_{2}(k)$ are :

$$
M_{1}(k)=\frac{\rho_{\mathrm{s} 1}}{k \tanh k h_{1}}, M_{2}(k)=\frac{\rho_{\mathrm{s} 2}}{k \tanh k h_{2}},
$$




\section{Horizons and Ergoregions in Superfluids}

where $h_{1}$ and $h_{2}$ are the thicknesses of the layers of two superfluids; $\rho_{\mathrm{s} 1}$ and $\rho_{\mathrm{s} 2}$ are superfluid densities of the liquids. Further we assume that the temperature is low enough so that the normal fraction of each of the two superfluid liquids is small, then $\rho_{\mathrm{s} 1} \approx \rho_{1}$ and $\rho_{\mathrm{s} 2} \approx \rho_{2}$.

Experiments with the $\mathrm{AB}$ interface ${ }^{9}$ where conducted in the 'deep water' limit $k h_{1} \gg 1$ and $k h_{2} \gg 1$. In the opposite limit of a thin slab, where $k h_{1} \ll 1$ and $k h_{2} \ll 1$, one obtains

$$
\alpha_{1}\left(\omega-\mathbf{k} \cdot \mathbf{v}_{1}\right)^{2}+\alpha_{2}\left(\omega-\mathbf{k} \cdot \mathbf{v}_{2}\right)^{2}=c^{2} k^{2},
$$

where

$$
\alpha_{1}=\frac{h_{2} \rho_{1}}{h_{2} \rho_{1}+h_{1} \rho_{2}}, \alpha_{2}=\frac{h_{1} \rho_{2}}{h_{2} \rho_{1}+h_{1} \rho_{2}}, c^{2}=\frac{F h_{1} h_{2}}{h_{2} \rho_{1}+h_{1} \rho_{2}} .
$$

This can be rewritten in the Lorentzian form (20) with the following effective contravariant metric $g^{\mu \nu}$ :

$$
g^{00}=-1, g^{0 i}=-\alpha_{1} v_{1}^{i}-\alpha_{2} v_{2}^{i}, g^{i j}=c^{2} \delta^{i j}-\alpha_{1} v_{1}^{i} v_{1}^{j}-\alpha_{2} v_{2}^{i} v_{2}^{j} .
$$

\subsection{Interaction with Environment}

The spectra (17) and (23) are valid for the perfect fluid, where dissipation due to friction and viscosity is neglected. They must be modified when the dissipation is added. For the ripplons propagating at the interface between two superfluids the dissipation leads to a simple extra term on the right-hand side of Eq.223. 211.

$$
M_{1}(k)\left(\omega-\mathbf{k} \cdot \mathbf{v}_{1}\right)^{2}+M_{2}(k)\left(\omega-\mathbf{k} \cdot \mathbf{v}_{2}\right)^{2}=F+k^{2} \sigma-i \Gamma \omega .
$$

For the ripplons at the interface between ${ }^{3} \mathrm{He}-\mathrm{A}$ and ${ }^{3} \mathrm{He}-\mathrm{B}$ the friction parameter $\Gamma>0$ depends on temperature and is proportional to $T^{3}$ at low $T^{22}$. The important property of the added dissipative term is that it introduces the reference frame of the environment. The $\omega$-dependence of the dissipative term in Eq. (28), which has no Doppler shift, implies that this spectrum is written in the frame of the container.

Since the main effect of this term is the introduction of the distinguished reference frame, the explicit form of this term is not important. That is why we expect that similar dissipative interaction with the environment exists for ripplons propagating on the free surface of the superfluid and even on the surface of normal viscous liquid:

$$
M(k)(\omega-\mathbf{k} \cdot \mathbf{v})^{2}=F+k^{2} \sigma-i \Gamma \omega .
$$

The parameter $\Gamma$ can be considered as phenomenological parameter, which depends on $\omega, k$ and the Reynolds number of the flowing liquid. 


\section{G.E. Volovik}

\subsection{Instability in the Ergoregion and Landau Criterion}

In superfluids the stationary (time-independent) flow of superfluid component is frictionless until the critical velocity of flow is reached. This is the essence of the phenomenon of superfluidity. However, the notion of critical velocity implies that there is a preferred reference frame with respect to which the velocity is counted. This is the reference frame of the environment (the frame of the container). In equations, this reference frame is provided by the $\Gamma$-term, which describes the dissipative interaction with the environment. This term also gives rise to the attenuation of ripplons.

When this term is taken into account, from the spectrum $\omega(k)$ in Eq. (29) it follows that the instability to the formation of the surface waves occurs when the velocity $v$ of the flow with respect to the wall exceeds the critical velocity $v_{L}$, at which the imaginary part $\operatorname{Im} \omega\left(k_{c}\right)$ of the energy spectrum of the critical ripplon with momentum $k_{c}$ crosses zero and becomes positive at $v>v_{L}$. At that moment the attenuation of ripplons transforms to amplification; and critical ripplons start to grow exponentially 211.

The critical velocity $v_{L}$ and the momentum of the critical ripplon $k_{c}$ do not depend on the friction parameter $\Gamma$. Moreover, at this velocity the real part $\operatorname{Re} \omega(\mathbf{k})=\omega^{\mathrm{com}}(k)+\mathbf{k} \cdot \mathbf{v}_{\mathrm{s}}$ also crosses zero: it becomes negative at $v>v_{L}$ (see Fig. 2 for ripplons at the AB interface, which we will discuss in Sec 3.5.). This means that the threshold velocity coincides with the Landau criterion for the ripplon nucleation:

$$
v_{L}=\min _{k} \frac{\omega^{\mathrm{com}}(k)}{k}, \omega^{\mathrm{com}}(k)=\sqrt{\left(\rho g+\sigma k^{2}\right) / M(k)} .
$$

The Landau critical velocity is different in the "relativistic" shallow-water and "non-relativistic" deep-water regimes:

$$
\begin{gathered}
v_{L}=c, \quad k_{c}=0, \quad \text { if } h k_{0}<\sqrt{3}, \\
v_{L}=c \sqrt{2 / h k_{0}}, \quad k_{c}=k_{0} \quad, \quad \text { if } h k_{0} \gg 1 .
\end{gathered}
$$

In both regimes the frequency of the critical ripplon is $\omega\left(k_{c}\right)=0$, i.e. the critical ripplon must be stationary in the wall frame.

The region in the liquid, where the flow velocity $v$ exceeds $v_{L}$, represents the ergoregion, since in the container frame the energy of the critical ripplon is negative in this region, $\omega^{\operatorname{com}}(k)+\mathbf{k} \cdot \mathbf{v}<0$. For the "relativistic" ripplons, the ergoregion is the region where $v$ exceeds $c$. This can be expressed in terms of the effective metric in Eq.(22): in the ergoregion the metric element $g_{00}$ changes sign and becomes positive. If the flow is perpendicular to the ergosurface (the boundary of the ergoregion), then the ergosurface serves as the event horizon for ripplons. It is the black hole horizon, if the liquid 


\section{Horizons and Ergoregions in Superfluids}

moves into the ergoregion, and it is the white hole horizon if the liquid moves from the ergoregion. However, the exponential growth of ripplons in the supercritical region is universal and does not depend on direction of the flow in the vicinity of the ergosurface. The flow can represent the white-hole or black-hole horizons; the relativistic ergoregion without the horizon; and even the ergogerion for the non-relativisic quasiparticles.

The instability develops due to the interaction with the fixed reference frame and occurs in the region where the energy of the critical fluctuation is negative in this frame. Such kind of instability is also called the Miles instability. ${ }^{231}$ In principle, Miles instability may take place behind the horizon of the atsronomical black holes if there exists the fundamental reference frame related for example with Planck physics 224. It may lead to the decay of the black hole much faster than the decay due to Hawking radiation.

\subsection{Ergoregion Instability vs Kelvin-Helmholtz Instability}

In the case of the interface between ${ }^{3} \mathrm{He}-\mathrm{A}$ and ${ }^{3} \mathrm{He}-\mathrm{B}$, the critical velocity of instability towards the growth of critical ripplon has been measured in the nonrelativistic deep-water regime ${ }^{9}$, and has been found in a good agreement with the theoretical estmate of the Landau velocity (modified for the case of two liquids 211) wihout any fitting parameter (see Ref. ${ }^{25}$ where also the turbulence triggered in bulk ${ }^{3} \mathrm{He}-\mathrm{B}$ by the interface instabilty is discussed). The important additional feature, which takes place in case of the interface between two sliding superfluids, is the existence of two different critical velocities.

One of them is the Landau critical velocity, above which the ergoregion appears for ripplons. In the typical situation the A-phase is stationary with respect to the walls of container, while the B-phase is moving with respect to the container with the velocity $v_{s B}$. For the deep-water regime and in the limit of low temperature $T \ll T_{c}$, this critical velocity is given by:

$$
v_{L}^{2}=\frac{2}{\rho} \sqrt{F \sigma} .
$$

The second critical velocity occurs if one completely neglects the interaction with the enviroment putting the friction parameter $\Gamma=0$. In this case the preferred reference frame disappears from the equations, and thus the physics is determined by the relative velocity $\mathbf{v}_{1}-\mathbf{v}_{2}$ of two superfluids. In this case it is well known that the Kelvin-Helmholtz instability of the interface occurs when the velocity difference reaches some threshold value. In our case, when the A-phase is stationary with respect in the container frame, while the B-phase is moving with respect to the container with the 


\section{G.E. Volovik}

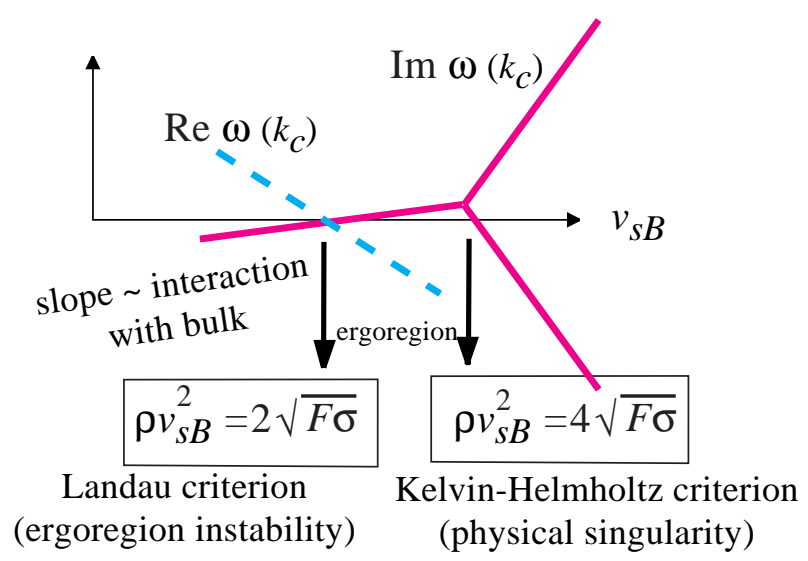

Fig. 2. Two critical velocities for ripplons living at the interface between two sliding superfluids - A and B phases of ${ }^{3} \mathrm{He}$. The lowest one is the Landau critical velocity, at which the energy of ripplons first becomes negative. The region with negative energy is called the ergoregion. If the friction parameter $\Gamma$ describing interaction with the environment is non-zero, the imaginary part of the frequency becomes positive in the ergoregion, i.e. attenuation of ripplons transforms to the amplification in the ergoregion which indicates the instability with increment proportional to $\Gamma$. The second velocity marks the more crucial Kelvin-Helmholtz instability at which the imarginary parts of both signs emerge. In the 'relativistic' shallow-water regime this velocity corresponds to physical singularity in metric field (Fig. 3). The shown values of two critical velocities correspond $T \ll T_{c}$, deep-water limit, and the experimental condition for the flow of two superfluids: the A-phase is stationary with respect to the walls of container, and the B-phase is moving with respect to the container with the velocity $v_{s B}$.

velocity $v_{s B}$, the relative velocity of two superfluids is $\left|\mathbf{v}_{1}-\mathbf{v}_{2}\right|=v_{s B}$ and the Kelvin-Helmholtz criterion becomes

$$
v_{K H}^{2}=\frac{4}{\rho} \sqrt{F \sigma} .
$$

Here we took into account that the densities of two liquids are equal $\rho_{1}=$ $\rho_{2}=\rho$. The difference between two critical velocities in such an arrangement is $\sqrt{2}$.

The behavior of the real and imaginary parts of the spectrum of the crtitical ripplon as functions of $v_{s B}$ is shown in Fig. 2. When $\Gamma \neq 0$, at $v_{K H}$ one has the smooth crossover, which becomes the true threshold at $\Gamma=0$. In the "relativistic" shallow-water regime, both thresholds have physical meaning, especially when the flow is radial (Fig. [3). The circumference 
Horizons and Ergoregions in Superfluids

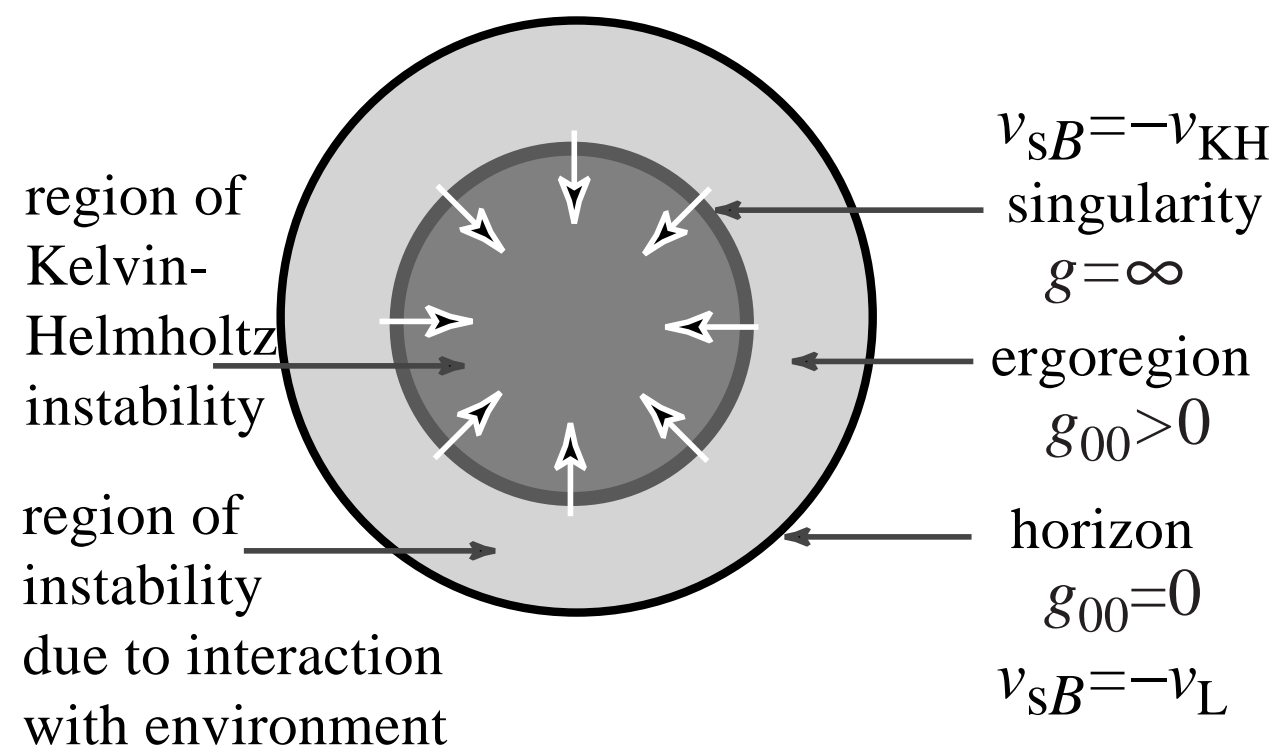

Fig. 3. Artificial $2+1$ black hole for ripplons at the $A B$ interface in the "relativistic" shallow-water limit, which can occur for the radial flow of the B-phase near the interface. The black hole horizon occurs when the flow reaches the Landau critical velocity $|v(r)|=v_{L}$. At larger critical velocity $|v(r)|=v_{K H}>v_{L}$ the Kelvin-Helmholtz instability occurs, which in terms of effective metric corresponds to the physical singularity within the black hole.

$v(r)=-v_{L}=-c / \sqrt{\alpha_{1}}$ represents the black-hole horizon. Deep inside the black hole, at $v(r)=-v_{K H}=-c / \sqrt{\alpha_{1} \alpha_{2}}$, the real singularity takes place: the $g_{r r}$ and $g_{0 r}$ components become infinite together with the determinant $g$ of the metric $g_{\mu \nu}$.

\section{HYDRAULIC JUMP AS WHITE HOLE}

\subsection{Hydraulic Jump}

The analog of a white hole horizon for the 'relativistic' ripplons on the surface of a shallow liquid is achieved in the kitchen-bath experiments. This is the so-called hydraulic jump first discussed by Rayleigh in terms of the shock wave 26. The circular hydraulic jump occurs when the vertical jet of water falls on a flat horizontal surface (Fig. 4). The flow of the liquid at the surface exhibits a ring discontinuity at a certain distance $r=R$ from 


\section{G.E. Volovik}

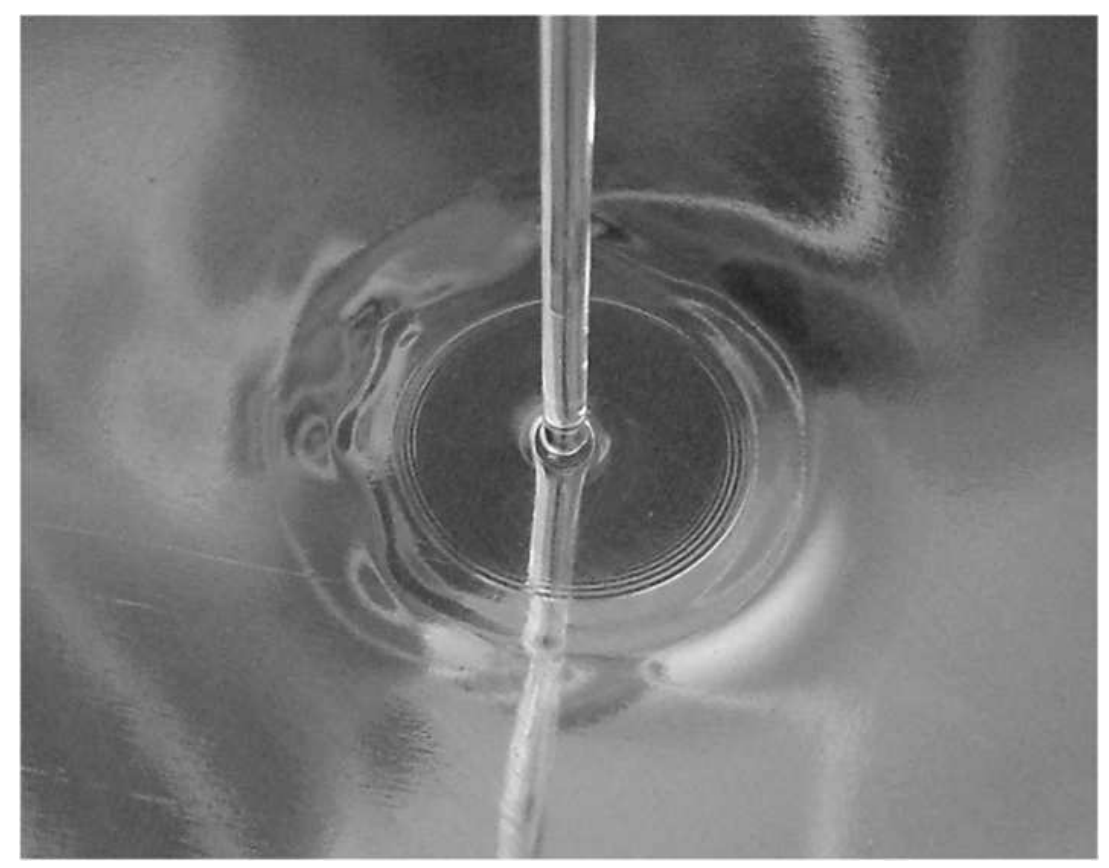

Fig. 4. Hydraulic jump. The standing waves at the otherwise smooth surface inside the white hole is the result of the egroregion instability. Courtesy Piotr Pieranski

the jet (observation of the non-circular hydraulic jumps with sharp corners has been reported in Ref. ${ }^{27}$ ). At $r=R$ there is an abrupt increase in the depth $h$ of the liquid (typically by order of magnitude) and correspondingly a decrease in the radial velocity of the liquid. The velocity of the liquid in the interior region $(r<R)$ exceeds the speed of 'light' for ripplons $v>c=\sqrt{h g}$, while outside the hydraulic jump $(r>R)$ one has $v<c=\sqrt{h g}$ (Fig. [5). Since the velocity flow is radial and outward, the interior region imitates the 'white-hole' region. The interval of the $2+1$ dimensional effective space-time in which the "relativistic" ripplons "live" is

$$
d s^{2}=-c^{2} d t^{2}+(d r-v(r) d t)^{2}+r^{2} d \phi^{2} .
$$

The surface inside the white hole is smooth, since all the perturbations are removed by 'superluminal' flow of the liquid. In general relativity the metric is continuous across the horizon. In our case there is a real physical singularity at the white-hole horizon - the jump in the effective metric (22). However, the discussed mechanism of the Miles instability in the ergoregion (or behind the horizon) discussed in Sec. 3.4. towards the growth of the critical ripplon does not depend on whether the horizon or ergosurface is 


\section{Horizons and Ergoregions in Superfluids}

smooth or singular. According to this instability the critical ripplon must be stationary in the wall frame. The standing waves inside the white hole are clearly seen in Fig. 4. The exponential growth of the critical ripplon is saturated by the non-linear effects, and then the whole pattern remains stationary (time-independent but not static).

A similar 3D analog of the black or white hole with the physical singularity at the horizon has been discussed by Vachaspati 28. The role of the physical horizon is played by the phase boundary between two quantum vacua - two superfluid phases of the same liquid, such as ${ }^{3} \mathrm{He}-\mathrm{A}$ and ${ }^{3} \mathrm{He}-$ B. Two vacua have different 'speed of light', say, different speed of sound, $c_{1} \neq c_{2}$. The analog of black or white horizon occurs if the superfluid velocity of the flow through the phase boundary is subsonic in one of the superfluids but supersonic in the other one, $c_{1}<v<c_{2}$.

\subsection{Hydraulic Jump in Superfluids}

The analogy between the instability of the surface inside the hydraulic jump and the instability of the vacuum behind the horizon can be useful only if the liquid simulates the quantum vacuum. For that, the liquid must be quantum, and its flow should not exhibit any friction in the absence of a horizon. That is why the full analogy could occur if one uses either the flow of quantum liquid with high Reynolds number, or the superfluid liquid which has no viscosity. Quantum liquids such as superfluid or normal ${ }^{3} \mathrm{He}$ and ${ }^{4} \mathrm{He}$ are good candidates.

The first observation of the circular hydraulic jump in superfluid liquid (superfluid ${ }^{4} \mathrm{He}$ ) was reported in Ref. 12]. The surface waves generated in the ergoregion (in the region inside the jump in Fig. 51) were also observed. As in the normal liquid, this instability is saturated by the non-linear terms. The same instability observed at the interface between A-phase and B-phase has a different consequence 9 : the instability is not saturated and leads to the crucial rearrangement of the vacuum state: quantized vortices start to penetrate into the ${ }^{3} \mathrm{He}-\mathrm{B}$ side from ${ }^{3} \mathrm{He}-\mathrm{A}$ [10. They partially or fully screen the ${ }^{3} \mathrm{He}-\mathrm{B}$ flow and reduce its velocity back below the threshold for the ripplon formation. Thus the instability kills the ergoregion.

Under the conditions of experiment 12 the hydraulic jump in superfluid

${ }^{4} \mathrm{He}$ is very similar to that in the normal liquid ${ }^{4} \mathrm{He}$. The position $R$ of the hydraulic jump as a function of temperature does not experience discontinuity at the superfluid transition. This suggests that quantized vortices are present in the flow providing the mutual friction between the superfluid and normal components of the liquid. As a result even below the $\lambda$-point, the 


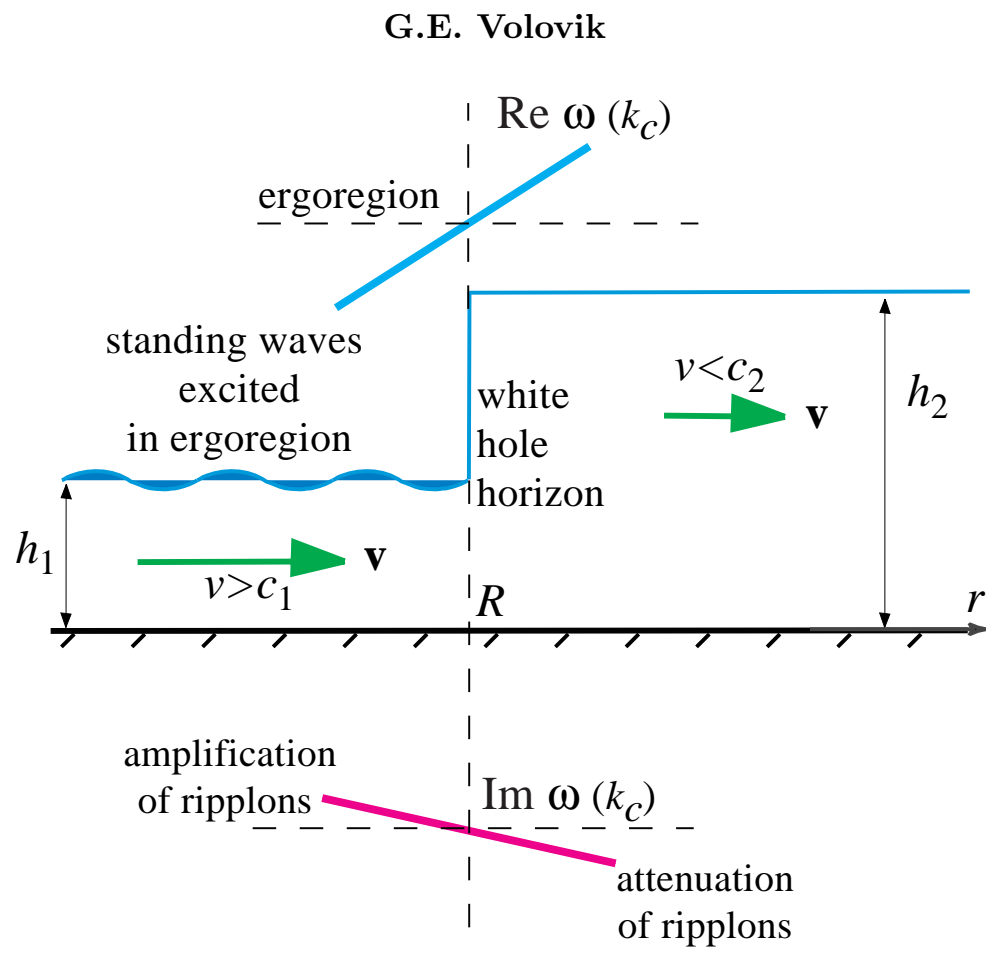

Fig. 5. Instability in the ergoregion. At the horizon both the energy and imaginary part of the ripplon spectrum cross zero. In the ergoregion where the energy is negative, the imaginary part is positive indicating instability towards the growth of the critical ripplon.

liquid moves as a whole though with lower viscosity because of the reduced fraction of the normal component.

To avoid the effect of the normal component it would be desirable to reduce the temperature or to conduct similar experiments in a shallow superfluid ${ }^{3} \mathrm{He}$.

The advantage of superfluid ${ }^{3} \mathrm{He}$ is that, as distinct from the superfluid ${ }^{4} \mathrm{He}$, vortices are not easily formed there: the energy barrier for vortex nucleation in ${ }^{3} \mathrm{He}-\mathrm{B}$ is about $10^{6}$ times bigger than temperature ${ }^{29}$. In addition, in superfluid ${ }^{3} \mathrm{He}$ the normal component of the liquid is very viscous compared to that in superfluid ${ }^{4} \mathrm{He}$. In the normal state the kinematic viscosity is $\nu \sim 10^{-4} \mathrm{~cm}^{2} / \mathrm{s}$ in liquid ${ }^{4} \mathrm{He}$, and $\nu \sim 1 \mathrm{~cm}^{2} / \mathrm{s}$ in liquid ${ }^{3} \mathrm{He}$. That is why in many practical arrangements the normal component in superfluid ${ }^{3} \mathrm{He}$ remains at rest with respect to the reference frame of the wall and thus does not produce any dissipation if the flow of the superfluid component is sub-critical.

One can also exploit thin films of a superfluid liquid, where the normal 


\section{Horizons and Ergoregions in Superfluids}

component is fixed. The ripplons there represent the so-called third sound (recent discussion on the third sound propagating in superfluid ${ }^{3} \mathrm{He}$ films can be found in Ref. ${ }^{30}$ ). In 1999 Seamus Davis suggested to use the third sound in superfluid ${ }^{3} \mathrm{He}$ for simulation of the horizons 31 .

In normal liquids it is the viscosity which determines the position $R$ of the hydraulic jump (see ${ }^{32}$ ). The open question is what is the dissipation mechanism which determines the position $R$ of the white-hole horizon in a superfluid flow with stationary or absent normal component when its viscosity is effectively switched off. Since there is no dissipation of the superfluid flow if its velocity is below $v_{L}$, one may expect that the same mechanism, which is responsible for dissipation in the presence of the horizon, also determines the position $R$ of the horizon. If so, the measurement of $R$ as function of parameters of the system will give the information on various mechanisms of decay of white hole. It is also unclear whether it is possible to approach the limit of a smooth horizon, without the shock wave of the hydraulic jump; and whether it is possible to construct the inward flow of the liquid which would serve as analog of the black hole horizon.

\section{CONCLUSION}

It appears that the ripplons - gravity-capillary waves on the surface of liquids or at the interfaces between superfluids - are the most favourable excitations for simulation of the effects related to horizons and ergoregions. The white-hole horizon for the "relativistic" ripplons at the surface of the shallow liquid is easily simulated using the kitchen-bath hydraulic jump. The same white-hole horizon is observed in quantum liquid - superfluid ${ }^{4} \mathrm{He}$ 12. The ergoregion for the "non-relativistic" ripplons is generated in the experiments with two sliding superfluids 9 .

The common property experienced by the ripplons in all these cases is the Miles instability inside the ergoregion or horizon. In some cases these instability is saturated leading to the standing waves inside the horizon or ergoregion, while in the other cases, such as for ripplons at the interface between ${ }^{3} \mathrm{He}-\mathrm{A}$ and ${ }^{3} \mathrm{He}-\mathrm{B}$, the instability leads to the complete elimination of the ergoregion. Because of the universality of the Miles instability, one may expect that it could take place inside the horizon of the astrophysical black holes, if there is a preferred reference frame which comes from the trans-Planckian physics (see Sec. 32.4 in Ref. ${ }^{2}$ ). If this is the case, the black hole would evapotate much faster than due to the Hawking radiation.

I hope, the future experiments in quantum liquids will explore the quantum limit, where the quantum effects related to horizon and ergoregion be- 


\section{G.E. Volovik}

come more pronounced. Experiments with hydraulic jump in superfluid ${ }^{4} \mathrm{He}$ must be extended to the low-temperature region $T \ll T_{c}$, while the experiments with ripplons at the interface between ${ }^{3} \mathrm{He}$ superfluids must be extended to the 'shallow-water' regime, where ripplons become 'relativistic'. And, of course, ripplons at the interface between different superfluid states of ultra-cold Bose and Fermi gases shall be used.

If the vacuum instability inside the horizon/ergoregion is saturated as in experiments with a free surface ${ }^{12}$, the other mechanisms will intervene such as the black-hole laser ${ }^{33}$, and even the quantum mechanical Hawking radiation of ripplons. The latter should be enhanced at the sharp discontinuous horizon of the hydraulic jump and maybe near the sharp corners of the non-circular (polygonal) hydraulic jump observed in Ref. 27].

\section{ACKNOWLEDGMENTS}

I thank Etienne Rolley and Michael Pettersen who sent me their experimental results prior to publication, Marc Rabaud and Tomas Bohr for discussions and Piotr Pieranski for the beautiful photo of the hydraulic jump. This work is supported in part by the Russian Ministry of Education and Science, through the Leading Scientific School grant \#1157.2006.2, and by the European Science Foundation COSLAB Program.

\section{REFERENCES}

1. S.A. Hughes, Trust but verify: The case for the atsrophysical black holes, hep-ph/0511217 R. Narayan, Black holes in astrophysics, gr-qc/050607; P.T. Chrusciel, Lect. Notes Phys. 604, 61 (2002).

2. G.E. Volovik, The Universe in a Helium Droplet, Clarendon Press, Oxford (2003).

3. W.G. Unruh, Phys. Rev. Lett. 46, 1351 (1981).

4. K. Srinivasan and T.Padmanabhan, Phys. Rev. D 60, 24007 (1999).

5. G.E. Volovik, JETP Lett. 69, 705 (1999).

6. M.K. Parikh and F. Wilczek, Phys. Rev. Lett. 85, 5042 (2000).

7. C. Barcelo, S. Liberati and M. Visser, Living Rev. Rel. 8, 12 (2005).

8. R. Schützhold and W.G. Unruh, Phys. Rev. D 66, 044019 (2002).

9. R. Blaauwgeers, V.B. Eltsov, G. Eska, A.P. Finne, R.P. Haley, M. Krusius, J.J. Ruohio, L. Skrbek and G.E. Volovik, Phys. Rev. Lett. 89, 155301 (2002).

10. A.P. Finne, V.B. Eltsov, R. Hanninen, N.B. Kopnin, J. Kopu, M. Krusius, M. Tsubota and G.E. Volovik, Novel hydrodynamic phenomena in superfluid ${ }^{3} \mathrm{He}$, cond-mat/0606619

11. G.E. Volovik, JETP Lett. 75, 418 (2002) and JETP Lett. 76, 240 (2002). 


\section{Horizons and Ergoregions in Superfluids}

12. E. Rolley, C. Guthmann, M.S. Pettersen and C. Chevallier, AIP Conference Proceedings 850, 141 (2006), physics $/ 0508200$

13. G.E. Volovik, JETP Lett. 82, 624 (2005).

14. P. Painlevé, C. R. Acad. Sci. (Paris) 173, 677 (1921); A. Gullstrand, Arkiv. Mat. Astron. Fys. 16(8), 1(1922).

15. A.J.S. Hamilton and J.P. Lisle, The river model of black holes, gr-qc/0411060

16. G.E. Volovik, Exotic properties of superfluid ${ }^{3} \mathrm{He}$, World Scientific, Singapore (1992).

17. T.A. Jacobson and G.E. Volovik, Phys. Rev. D 58, 064021 (1998).

18. M. Nouri-Zonoz and T. Padmanabhan, The classical essence of black hole radiation, gr-qc/9812088

19. V.A. Belinski, Phys. Lett. A 354, 249 (2006).

20. E.T. Akhmedov, V. Akhmedova and D. Singleton, Hawking temperature in the tunneling picture, hep-th/0608098

21. S.W. Hawking, Comm. Math. Phys. 43, 199 (1975).

22. N.B. Kopnin, JETP 65, 1187 (1987).

23. R. Schützhold and W.G. Unruh, Phys. Rev. Lett. 95, 031301 (2005).

24. W.G. Unruh and R. Schtzhold Phys. Rev. D 71, 024028 (2005).

25. V.B. Eltsov, M. Krusius and G.E. Volovik, Transition to superfluid turbulence, J. Low Temp. Phys. this issue; cond-mat/0608537.

26. L. Rayleigh, Proc. Roy. Soc. Lond. A 90, 324 (1914).

27. C. Ellegaard, A.E. Hansen, A. Haaning, K. Hansen, A. Marcussen, T. Bohr, J.L. Hansen, and S. Watanabe, Nature 392, 767 (1998).

28. T. Vachaspati, J. Low Temp. Phys. 136, 361 (2004).

29. Ü. Parts, V.M.H. Ruutu, J.H. Koivuniemi, Yu. N. Bunkov, V.V. Dmitriev, M. Fogelström, M. Huenber, Y. Kondo, N.B. Kopnin, J.S. Korhonen, M. Krusius, O.V. Lounasmaa, P.I. Soininen and G.E. Volovik, Europhys. Lett. 31, 449 (1995).

30. A. Vorontsov and J.A. Sauls, J. Low Temp. Phys. 134, 1001 (2004).

31. S. Davis, private communication at NEDO 2nd International Workshop on Quantum Fluids and Solids, Hawaii, January 1999.

32. T. Bohr, P. Dimon and V. Putkaradge, J. Fluid Mech. 254, 635 (1993); T. Bohr, C. Ellegaard, A.E. Hansen and A. Haaning, Physica B 228, 1 (1996).

33. S. Corley and T. Jacobson, Phys. Rev. D 59, 124011 (1999). 